EP-99

\title{
Skewing of Sodium Antimony Gluconate mediated therapy for a predominant Th1 during visceral leishmaniasis on triggering CD2 epitope to circumvent liver infection
}

\author{
Sukrat SINHA*
}

Department of Zoology, Nehru Gram Bharati, Prayagraj, India

Introduction: Visceral leishmaniasis (VL) is a macrophage associated disorder which is linked with a profound decrease in the immunotherapeutic potential of the infected subjects leading to a marked reduction in the CD4 linked Th1 protective immune response. It greatly affects the liver leading to abnormal levels of serum glutamic pyruvic transaminase and serum glutamic oxaloacetic transaminase. Also the patients suffering from VL have been reported to be coinfected with Hepatitis C during some circumstances. Simultaneously the patients in Bihar are showing unresponsiveness towards sodium antimony gluconate which is still a first line of drug in many countries around the world against VL.

Methods: The T cells were isolated from patients and were subjected to fluroscence associated cell sorting analysis and cytokine analysis.

Results: We have reported down regulation of CD2 co receptor on the surface of CD4 cells in patients suffering from VL. Stimulation of CD2 epitope with anti CD2 antibody has led to a remarkable increase in the protein kinase C alpha mediated phosphorylation on $\mathrm{CD} 2$ co receptor on CD4 T cells, induction of interferon (IFN)- $\gamma$ led Th1 dominated immune response, a substantial increase in the lymphoblast population and this response remained Th1 dominated even in the presence of Th2 predominant conditions signified with recombinant interleukin-4.

Conclusions: We have shown that biological immunomodulators such as IFN- $\gamma$ can provide a missing signal and enhance the activity of antimonials in the treatment of VL and cutaneous leishmaniasis. 